\title{
MEASUREMENT OF INUNDATING FLOW FROM A BROKEN ENBANKMENT BY USING VIDEO IMAGES SHOOT FROM A MEDIA HELICOPTER
}

\author{
Ichiro Fujita ${ }^{1, *}$, Yuichi Notoya ${ }^{2}$, and Takanori Furuta ${ }^{3}$ \\ ${ }^{1}$ Department of Civil Engineering Kobe University, 1-1 Rokkodai, Nada-ku, Kobe, Japan \\ ${ }^{2}$ CTI Engineering Co., Ltd., 1-6-7 Doshomachi, Chuo-ku, Osaka, Japan \\ ${ }^{3}$ Graduate School of Engineering, Kobe University, 1-1 Rokkodai, Nada-ku, Kobe, Japan
}

\begin{abstract}
The heavy rain disaster in the Kinugawa River basin that occurred along with the passage of the Typhoon 18 caused the embankment destruction in the middle reach of the river on September 10, 2015. Due to the overflow, the houses in the vicinity of the embankment collapsed, causing a flood inundation spreading over a wide area. Because the embankment breakwater occurred during the daytime, the state of the inundating flow was recorded from various angles by media helicopters or drones. In this study, we developed a method to extract quantitative flow information from a helicopter video image in which the shooting position and angles are changed one after another, because it was taken in emergency. In the analysis, the images were orthorectified after stabilizing the images, from which surface velocity distributions were measured by image-based technique such as the large-scale particle image velocimetry (LSPIV) or the space-time image velocimetry (STIV). As a result, the time change of water entering from the broken embankment and the total inundated water volume during the disaster were estimated. In addition, two-dimensional surface velocity distributions were analysed to show the spreading of inundated flow.
\end{abstract}

\section{Introduction}

At the time of a river disaster, the state of the disaster is sometimes observed from a media helicopter and broadcasted from the site. In another case, images taken from a camcorder or an UAV by a local resident are uploaded on the web site to widely notify the actual situation of the disaster. The advantage of using airborne images is that they can capture a wide area of the disaster safely from the air without any difficulty. The point to note in such a condition is that the images are obtained not with static photographs but with movies, which can convey the situation of disaster more vividly. Moreover, such images could be used to extract quantitative information of the disaster especially when the water-related disaster is concerned because surface flow velocity distributions can be measure using

\footnotetext{
*Corresponding author: ifujita@kobe-u.ac.jp
} 
image-based techniques such as the large-scale particle image velocimetry (LSPIV) $[1-5,10]$ or the space-time image velocimetry (STIV)[6-10]. As for the usage of web-uploaded image, Boursicaud et al. [11] analysed a debris flow captured from a bridge by a local resident. However, images captured by a media helicopter have never been analysed in the past researches.

In this paper, an image analysis system for measuring water surface flow was developed that can treat airborne video images in which the shooting angle varies from time to time. The target disaster occurred on September 10, 2015 along with the passage of the Typhoon 18 in the Kinugawa River basin in Japan. In this disaster, the embankment of the Kinugawa River was destructed by the overtopping flow from the main channel in the middle reach of the river and the inundated flow destroyed the houses in the vicinity of breach section. Moreover, the extended inundation caused flooding on the floor of many houses within a wide residential area. Figure 1 shows a general view of the inundated area caused by the breakdown of the left bank. The main factor of the disaster was that a linearly extended precipitation band remained just above the long river reach of the Kinugawa River for several hours and caused a significant increase in the river discharge. Since the embankment breakdown occurred during the daytime, the state of the inundation was clearly observed by a media helicopter and broadcasted for a long time. In this research, five DVDs were provided by the Japan Broadcasting Corporation that recorded three and a half hour video of the disaster, including the overflowing flow from the broken part of the embankment, wooden houses being destroyed by the flow, and spreading of inundated flows. Although most of the field of view of the video images changes from frame to frame, a few seconds of scenes with little background movement was searched for and they were analysed by applying the developed image analysis system.
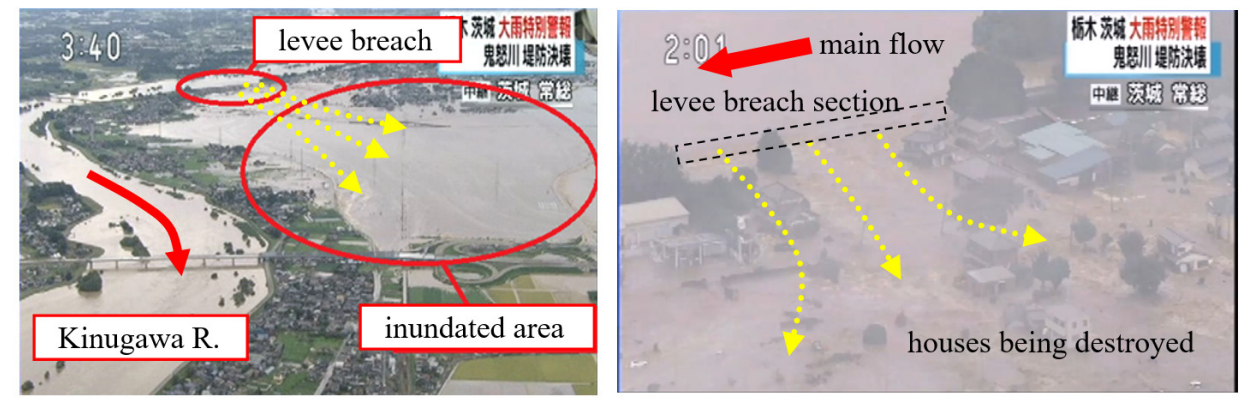

Fig 1. Location of levee breach and inundated area.

\section{Outline of the provided video}

The video provided by the media was compressed in size probably due to the copyright restriction, i.e. the image size provided was 720 by 480 pixels of the VOB file type, not the original high definition size of 1920 by 1080 pixel. In addition, some subtitle letters were superimposed on the image, deteriorating the quality for the purpose of image analysis. What was offered by the Japan Broadcasting Corporation was a movie broadcasted on September 10, 2015, which were recorded on 5 DVDs, covering time periods from 13:40 to around 18:00. The frame rate of the movie is $30 \mathrm{fps}$. Since the image was taken for the purpose of reporting the disaster, the helicopter turned around the site while shooting the image from various angles repeating zoom in and zoom out from time to time. Among the numerous scenes, five scenes were extracted that captured the surface flow overflowing from the breakdown part of the embankment. The upper-row photos in Table 1 shows the 
first frame images extracted. Although the captured images can provide valuable information with respect to the inundation process including the trees being flashed away and wooden houses being collapsed, the duration of image keeping the same viewing angle was very short and it made the surface flow measurement difficult to perform continuously. Therefore, even a scene capable to use for surface flow image analysis had merely a duration of about 1 second as indicated in Table 1, in which the middle-row image is the last frame image captured. However, even in the carefully extracted scenes, the background moves continuously little by little when the first and the last images are compared, so the image stabilization was necessary, which will be shown later. In addition, since the image shooting was basically conducted from an oblique direction, ortho-rectification of the image had to be applied to use for the imaging techniques.

Table 1. Major scenes used for the analysis at different times.

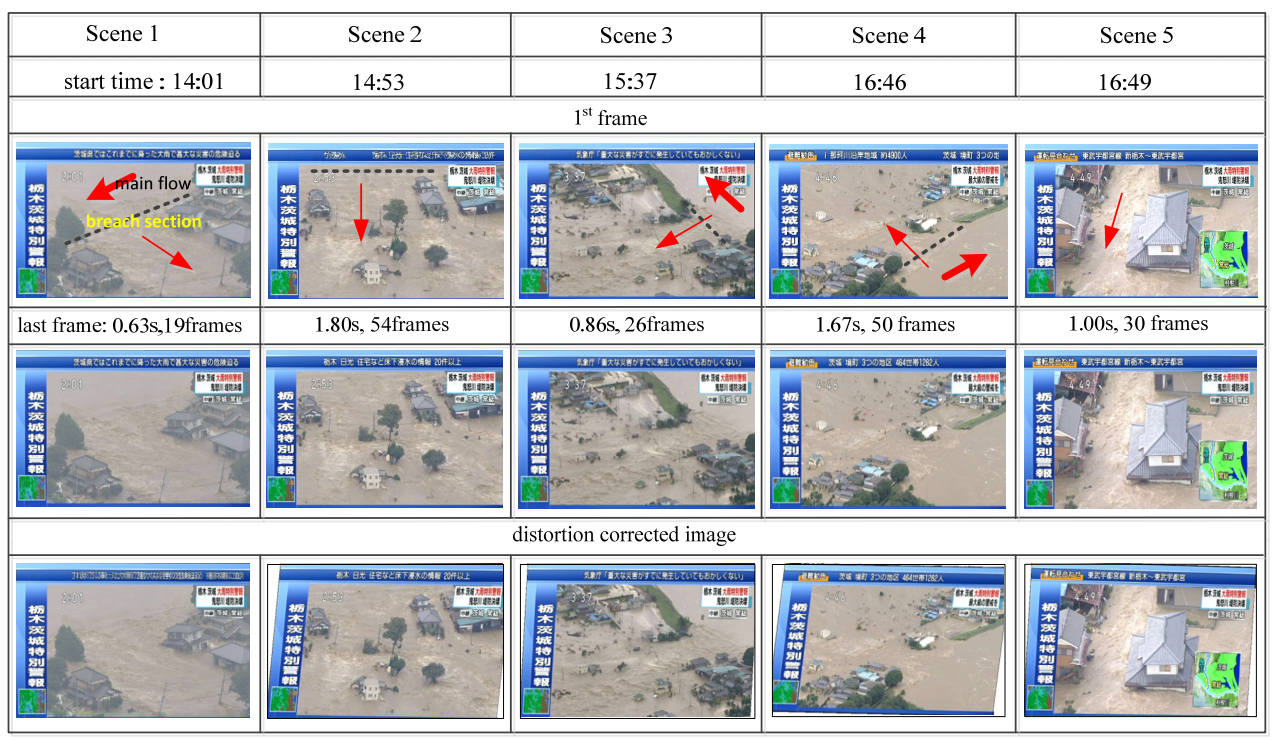

\section{Outline of the image process}

Before applying image-based techniques, the original unstable images were processed to yield ortho-rectified images by following the four steps indicated in Figure 2. In the first step, the background shift of sequential images were measured by applying the

\begin{tabular}{|c|c|c|c|c|c|c|}
\hline $\begin{array}{l}\text { Step } 1 . \\
\text { image stabilization: } \\
\text { use marker racking } \\
\text { method to obtain }\end{array}$ & $\Longrightarrow$ & $\begin{array}{l}\text { Step } 2 . \\
\text { picking up more } \\
\text { than six GCPs: } \\
\text { use detailed LP }\end{array}$ & $\Longrightarrow$ & $\begin{array}{l}\text { Step } 3 . \\
\text { estimation of } \\
\text { parameters for } \\
\text { image mapping }\end{array}$ & $\Longrightarrow$ & $\begin{array}{l}\text { Step } 4 . \\
\text { transformation } \\
\text { of distorted } \\
\text { images }\end{array}$ \\
\hline
\end{tabular}

Fig. 2 Image processing steps.

marker tracking (MK) method as indicated in Figure 3. A free software (Blender) is used in this step. Figure 3 provides an example of the background shift measurements by using MK method. MK method can measure the background shift vector by applying a technique similar to the particle image velocimetry (PIV). In the example shown in Figure 3, a lot of reference windows indicated by yellow squares are set manually on the positions of objects 
that are fixed to the ground such as houses. In Figure 3, a shearing deformation is detected and with this information the second image is deformed to coincide with the first image. This process is repeated for all the extracted frames indicated in Table 1. The third image row in Table 1 provides the deformed image for the last frame.
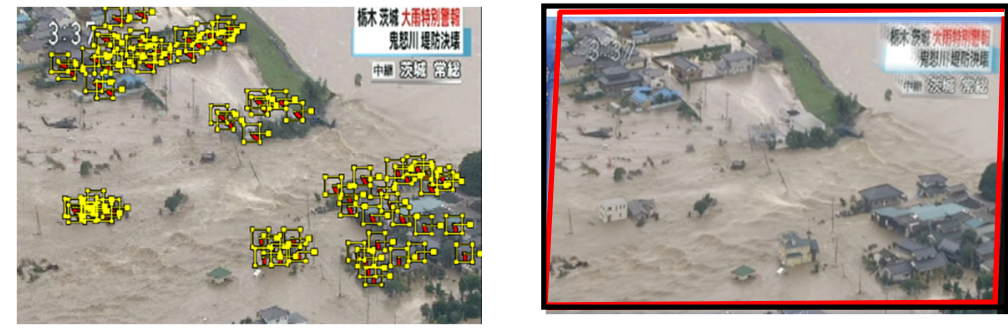

Fig.3 Application of marker tracking method. left : yellow square is the search window and red line is the shift vector, right : stabilized image.

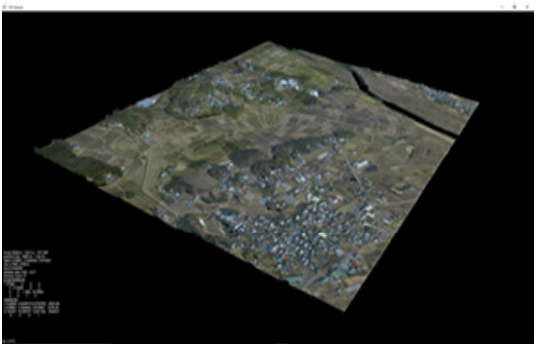

(a) 3D view of entire LP data

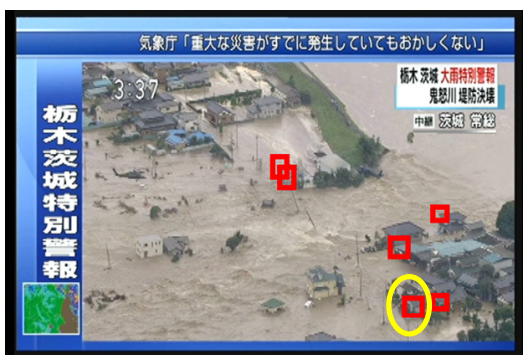

(c) setting of GCPs

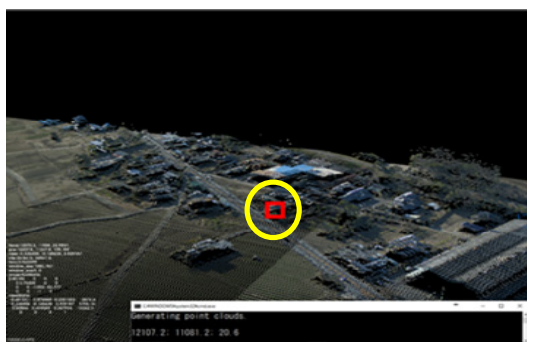

(b) 3D view of local LP data

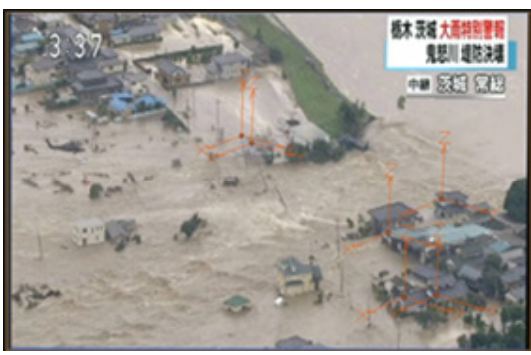

(d) local coordinates of GCPs

Fig.4 Picking up GCPs using LP data.

In the second step, three dimensional coordinates of more than six ground control points (GCPs) to use for the image rectification are obtained by using detailed LP data of the site. Specifically, the LP data is expressed as CG shown in Figure 4(a) and the detailed original measurement data is plotted as indicated in Figure 4(b). The GCPs are carefully selected at some fixed points such as the tip of a roof as shown in Figure 4(c) and their coordinates are obtained from the original LP data shown in Figure 4(b). For example, the position shown as a dotted circle in Figure 4(b) corresponds to the tip of one of the roof in Figure 4(c). The respective $3 \mathrm{D}$ coordinates of GCPs are obtained from the original LP data. The local coordinates for each GCP is shown in Figure 4(d).

In the third step, the parameters for transforming the stabilized obliquely viewed images to ortho-rectified images were estimated. The parameters are the camera coordinates in the 
air, the focal length of the lens, and the three camera angles (roll, pitch and yaw). These parameters were estimated in a trial and error manner so that the field of view of the obliquely viewed image generated by CG software agrees with the actual video image.

In the final step, the oblique image are transformed into an ortho-rectified image by using the software KU-STIV developed by authors's group $[12,13]$ by using the parameters

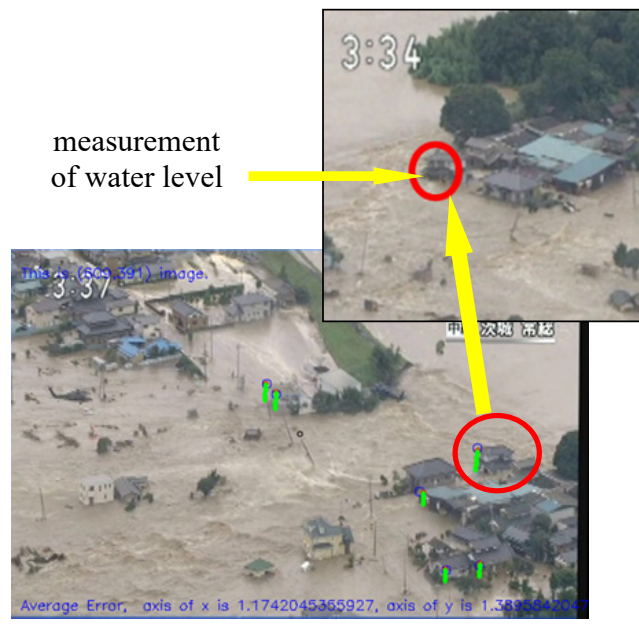

(a) stabilized original image

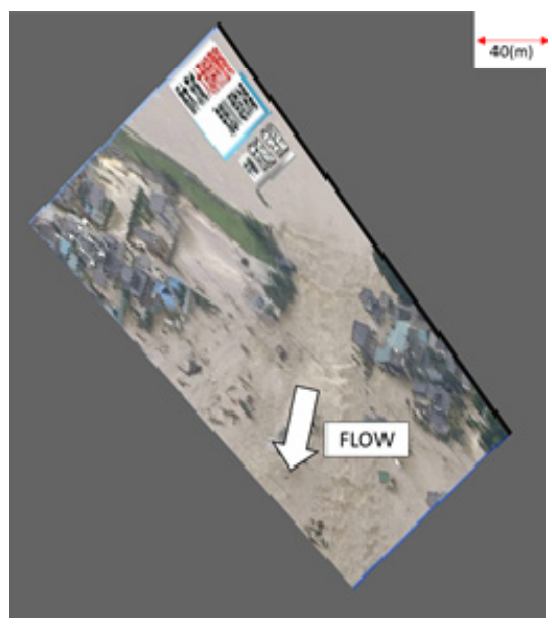

(b) ortho-rectified image

Fig.5 Image transformation of stabilized oblique image.

determined in the $3^{\text {rd }}$ step and the water level. In KU-STIV, a line segment drawn vertically from the GCP to the water level appears when the parameters for transformation is appropriate. Such vertical lines, green in colour, are shown in Figure 5(a). The water level was measured from an image viewed from another angle indicated in Figure 5. The orthorectified image is provided in Figure 5(b) demonstrating the overflow from the levee breach section.

\section{Image analysis of overflowing surface flow}

With the ortho-rectified images obtained so far, surface velocity distributions were obtained either by LSPIV or STIV depending on the measurement purposes. In addition, the inundating discharge hydrograph was estimated together with the time variation of embankment breakage width.

\subsection{Time variation of levee breach width}

According to the interview survey of local people, the levee breach started at 12:50 and the initial width is estimated to be about 20 meters. Other than the images indicated in Table 1 , there are several scenes in which the width of the breakage can be observed clearly. The physical scale of the width were estimated by comparing with the detailed LP data introduced in Figure 4. Figure 6 shows the time variation of the levee breach width. It is obvious that the width increased quickly for one hour after the breakdown and after that it increased almost linearly with time until it reached the maximum width of about 200 meters, causing a huge amount of inundated water into the residential area. 


\subsection{Estimation of overflowing discharge}

In order to estimate the overflowing discharge from the levee breach section, surface velocity distribution at the entering section was measured by the image-based technique STIV rather than LSPIV, because STIV is applicable for obtaining quantitative data even under deteriorated images such as the scenes provided in the present disaster. As an example, the measurement result by STIV at 15:37 is provided in Figure 7 together with some of the space time images (STIs). Although the texture appeared on an STI includes noisy patterns, an inclined pattern corresponding to the flow velocity is visible. In Figure 7, the texture on STI is enhanced by applying a histogram equalization filter. The average surface velocity is then calculated by taking the average of the total velocity data and it was

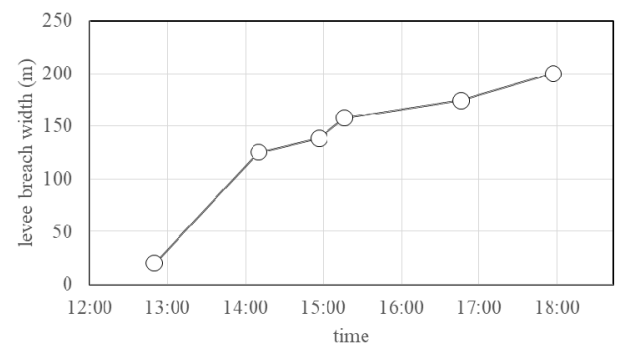

Fig. 6 Time variation of levee breach width.

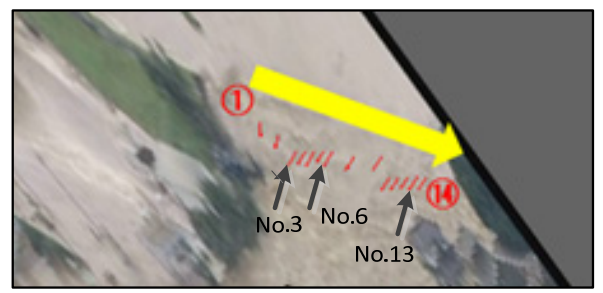

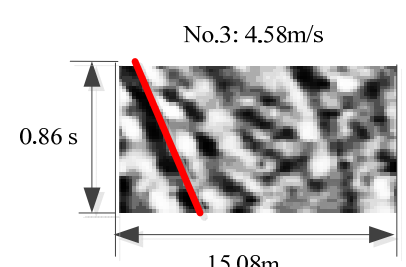

$15.08 \mathrm{~m}$

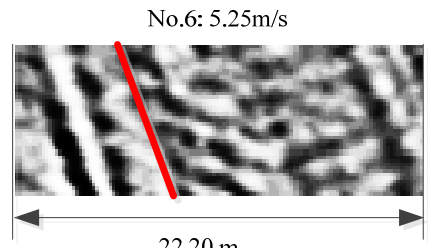

$22.20 \mathrm{~m}$

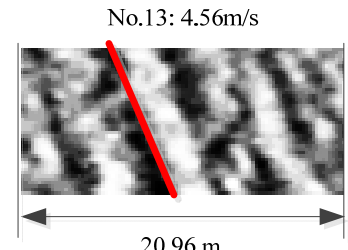

$20.96 \mathrm{~m}$

Fig. 7 STIV analysis for the scene 3 at $15: 37$.

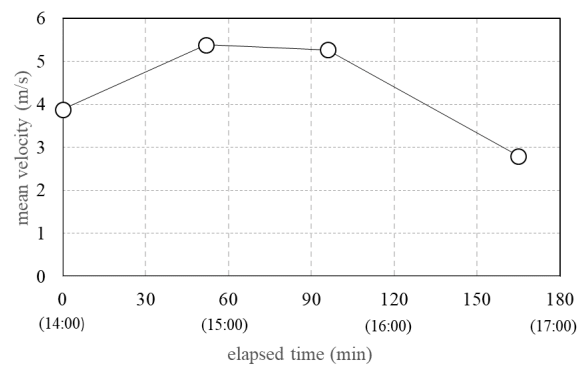

Fig. 8 Time variation of mean velocity outflowing from the levee breach section. 
$5.05 \mathrm{~m} / \mathrm{s}$ for the scene 3 at $15: 37$. The time variation of the outflowing mean velocity measured by analysing similar procedure for the other scenes is provided in Figure 8 . The mean velocity takes a peak value of about $5 \mathrm{~m} / \mathrm{s}$ after 15:00 and decreases gradually to less than $3 \mathrm{~m} / \mathrm{s}$ before $17: 00$.

By combining the time variation of the breach width shown in Figure 6, the outflowing mean velocity in Figure 8 and the estimated overflowing critical water depth, the evolution of accumulated inundated water volume is estimated as shown in Figure 9. The surface velocity coefficient that convert surface velocity to depth-averaged velocity was set at 0.85 in this case. According to the present calculation, the inundated water volume was estimated to be about $1.10 \times 10^{7} \mathrm{~m}^{3}$, which roughly agrees with the officially reported data.

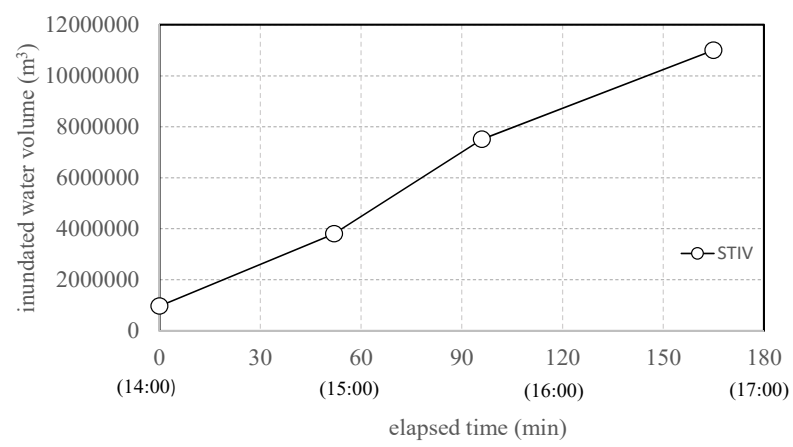

Fig. 9 Accumulated inundating water volume.

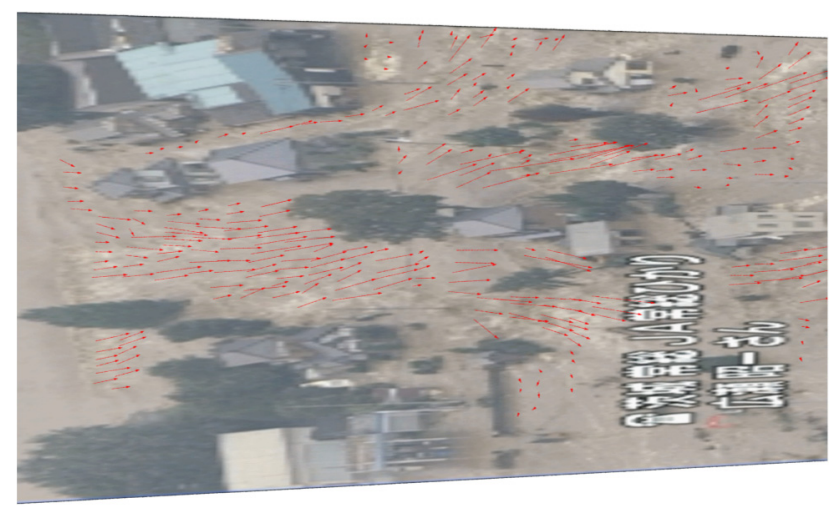

Fig. 10 Surface velocity distribution obtained by LSPIV for the scene 3.

\subsection{Two dimensional inundating flow}

In order to obtain the spreading flow features from the breach section, the large-scale particle image velocimetry (LSPIV) technique was applied to the ortho-rectified images. In the present analysis, a LSPIV software was developed so that the template for the pattern matching in LSPIV can be placed at an arbitrary point. This is because a template that contains a part of a house would yield erroneous values. Therefore, the templates were carefully set so that they include only water flowing area. Figure 10 provides an example of surface velocity distribution for the scene 3 obtained 13:47. The template size was set at 40 by 40 pixels with a physical dimension of 2.4 meters and 26 frame images were used to obtain the short time average data of 0.87 seconds. The result shows that the overflowing 
water was accelerated downstream of the breach section and the flow spreads widely in between the residential area. The vector field presented in Figure 10 can be used as a measurement data when an inundation simulation is executed in this area.

\section{Conclusions}

Conventionally, airborne video images of an inundation disaster have been used only to browse the inundating flow and have not been used to extract quantitative flow information. In the present research, such images usually with a large background movement were analysed by developing a novel image processing system. Although the quality of the video images provided by the broadcasting company was intentionally deteriorated due to the authorship issue, the developed system could successfully measure the spreading of the inundating flow by appropriately applying STIV or LSPIV. Measurement accuracy would be significantly improved if the original video images had been provided, but it should be noted that even with images of low quality the surface velocity analysis was shown to be possible when the field of view of the images were almost fixed at least for several seconds. The same thing can be said when using UAV for surveying a disaster.

The Japan Broadcasting Corporation provided valuable videos of the Kinugawa disaster and a financial support was given by the research fund of the River Foundation. We are grateful to their supports.

\section{References}

1. I. Fujita, S. Komura, Annual J Hydraul Eng JSCE, 38,733(1994)

2. I. Fujita, M. Muste, A. Kruger, J. Hydraul Res. 36, 397 (1998)

3. M. Muste, I. Fujita, A. Hauet, Water Resour Res, 40 (2008)

4. J. Le Coz, et al., J Hydrol, 394, 42 (2010)

5. N.F. Guillén, A. Patalano, C.M. García, J.C. Bertoni, Nat Hazards, 87, 383 (2017)

6. I. Fujita, H. Watanabe, R. Tsubaki, Int J River Basin Man, 5, 105 (2007)

7. I. Fujita, Y. Kunita, R. Tsubaki, Aust J Water Resour 16, 12 (2013)

8. I. Fujita,Water, 9, 269 (2017)

9. I. Fujita, et al., Journal of JSCE, 5, 305 (2017)

10. J. Aberle, C.D. Rennie, D.M. Admiraal, M. Muste, Experimental Hydraulics :Methods, Instrumentation, Data Processing and Management, Vol.II :Instrumentation and Measurement Techniques (CRC Press, 2017)

11. R. L. Boursicaud, L. Pénard, A. Hauet, F. Thollet, J.L. Coz. Hydrol. Process. 30, 90 (2016)

12. I. Fujita, et al., J JSCE, Ser.B1 (Hydraulic Engineering), 73, 4, I_499 (2017)

13. I. Fujita, et al. Proc 37th IAHR World Congress, 5284 (2017) 